\title{
MALDI-TOF MS for the rapid identification and drug susceptibility testing of filamentous fungi
}

\author{
YANG PENG $^{1}$, QIN ZHANG ${ }^{1}, \mathrm{CHAO}^{\mathrm{X}} \mathrm{U}^{2}$ and WEIFENG SHI ${ }^{1}$ \\ ${ }^{1}$ Department of Clinical Laboratory, The Third Affiliated Hospital of Soochow University, Changzhou, Jiangsu 213003; \\ ${ }^{2}$ Department of Clinical Laboratory, Shanghai Tenth People's Hospital Chongming Branch, \\ Chongming, Shanghai 202157, P.R. China
}

Received January 31, 2019; Accepted September 24, 2019

DOI: $10.3892 /$ etm.2019.8118

\begin{abstract}
The present study aimed to evaluate the applicability of matrix-assisted laser desorption/ionization time-of-flight mass spectrometry (MALDI-TOF MS) for identifying filamentous fungi and assessing the in vitro activities of common antifungal drugs against different kinds of filamentous fungi that are commonly encountered in a clinical setting. A total of 123 strains of filamentous fungi (24 species) were submitted for identification by MALDI-TOF MS, and the findings were compared with those obtained by conventional methods. The discrepancies were further investigated by internal transcribed spacer (ITS) sequence analysis. Then, 79 strains were randomly selected for further testing by the minimum inhibitory concentration Etest method. MALDI-TOF MS correctly identified $114(92.70 \%)$ of the 123 filamentous fungi and failed to identify six isolates (4.9\%). By contrast, the conventional identification methods made $113(91.9 \%)$ correct identifications. In addition, 15 isolates of filamentous fungi were further identified by ribosomal DNA-ITS sequencing. In the in vitro antifungal susceptibility test, voriconazole showed the strongest antifungal activity among the tested drugs against a broad range of filamentous fungi. Caspofungin showed a better in vitro antifungal activity than fluconazole, itraconazole, and amphotericin B. MALDI-TOF MS offers a cost/time-saving, high-throughput and accurate working protocol for identifying filamentous fungi. Voriconazole could still serve as the first-line drug for treating serious infections caused by filamentous fungi, while caspofungin may be another treatment option for fungal infections.
\end{abstract}

Correspondence to: Professor Weifeng Shi, Department of Clinical Laboratory, The Third Affiliated Hospital of Soochow University, 185 Juqian Road, Changzhou, Jiangsu 213003, P.R. China E-mail: shiweifeng67@163.com

Key words: matrix-assisted laser desorption/ionization time-of-flight mass spectrometry, filamentous fungi, internal transcribed spacer, Etest

\section{Introduction}

The increase in antimicrobial abuse, bone marrow and organ transplantation, invasive treatments, and incidences of HIV infections and cancer in the past decades has resulted in an increase in the incidence of mycoses due to emerging filamentous fungi (1). Most filamentous fungi grow slowly, therefore it often takes several days for the laboratory diagnosis of a fungal infection (2-4). Furthermore, some filamentous fungi, such as Sporothrix schenckii, Sporothrix globosa, Trichophyton interdigitale, Trichophyton mentagrophytes, Arthroderma benhamiae and Trichophyton rubrum cannot be clearly differentiated by microscopic examination after traditional culturing and staining. Their overlapping phenotypic characteristics can be confusing, and identification requires growth of the organisms in culture for at least one week, thus delaying the diagnosis (5-9).

It is crucial to develop rapid identification methods and effective treatments for infections by filamentous fungi (10). Molecular techniques such as PCR, ribosomal DNA (rDNA) internal transcribed spacer (ITS) sequence analysis, or $18 \mathrm{~S}$ ribosomal RNA (rRNA) gene sequencing can accurately identify many filamentous fungi at the species level (11). However, these methods are not only time-consuming and expensive but also require expertise $(12,13)$. Matrix-assisted laser desorption/ionization (MALDI) time-of-flight mass spectrometry (MALDI-TOF MS) offers a time-saving and highly accurate means for rapid identification of microbial agents, especially bacteria (14-17). In recent years, the application of MALDI-TOF MS has also emerged as a means to identify isolates from fungi, and it is a faster and more robust diagnostic technique compared with the other established protocols for the identification of yeasts isolated from carious clinical specimens $(18,19)$. After the successful MALDI-TOF MS identification of bacteria and yeasts in clinical laboratories, increased interest has emerged in applying this method to filamentous fungi $(20,21)$. There are however some disadvantages to this method. MALDI-TOF identification has been hindered by the absence of filamentous fungi from in-house MALDI-TOF MS libraries. Consequently, several isolates have been mis-identified or unidentified because of the absence of the important reference spectra in the databases $(22,23)$. Additionally, the high cost of equipment prevents this technique from being used in developing 
countries (24). In light of this, this present study attempted to identify 123 strains of filamentous fungi by using conventional methods and MALDI-TOF MS. When these methods gave discordant results, the discrepancies were further confirmed by ITS sequence analysis.

The Clinical and Laboratory Standards Institute has developed a reference broth (M38A2) microdilution (BMD) approach for testing the antifungal susceptibility of various filamentous fungi (25). Unfortunately, this method is cumbersome. By contrast, commercially available Etest strips containing defined concentration gradients of drugs provide a simple and easy approach for testing the susceptibility of filamentous fungi. In the present study, the Etest method was employed to investigate the antifungal activities of five antifungal agents (fluconazole, amphotericin B, voriconazole, itraconazole and caspofungin) against 79 strains of filamentous fungi in vitro.

\section{Materials and methods}

Fungal isolates. A total of 123 filamentous fungi were collected in this study. Of these 123 fungi, 19 standard strains were purchased from The China Medical Culture Collection Center (Table I). The remaining 104 isolates were from clinical specimens: 21 isolates were obtained from The Clinical Laboratory of the Third Affiliated Hospital of Soochow University, and the remaining 83 strains were kindly provided by the Clinical Laboratory of The Jiangsu Province Hospital and Nanjing General Hospital. These clinical isolates consisted of sputum, urine, bronchial washing fluid and skin.

Phenotypic identification. All standard strains and clinical isolates were subcultured on Sabouraud dextrose agar (SDA) at $28^{\circ} \mathrm{C}$ for 2- 6 days. The fungi were first identified by skilled mycologists on the basis of macroscopic and light microscopic features using conventional methods, including lactophenol cotton blue and $\mathrm{KOH}$ staining. Briefly, the size, shape, pigment, texture and growth conditions of each isolate were recorded. Subsequently, the spores and hyphae were smeared on glass slides, stained with lactophenol cotton blue (Baso Diagnostic, Inc.) or $10 \% \mathrm{KOH}$, and observed under an Olympus IX53 microscope at a final magnification of x1,000.

Identification by MALDI-TOF MS. Protein analysis was performed using a MALDITOF Microflex LT mass spectrometer with a $\mathrm{N} 2$ laser set at $337 \mathrm{~nm}$. The spectra were recorded in positive ion mode at a frequency of $60 \mathrm{~Hz}$ laser within a m/z range of 2,000 to 20,000. For each spectrum, a total of 240 shots from different positions of the metal plate were collected. The acceleration voltage, extraction voltage, lens voltage and delayed extraction time were set as $20 \mathrm{kV}$, $18.00 \mathrm{kV}, 6 \mathrm{kV}$ and $150 \mathrm{~ns}$, respectively. In addition,the flow rate was maintained at $70 \mathrm{l} / \mathrm{min}$. The fungal isolates were cultured and prepared for MALDI-TOF MS (Bruker Corporation) in accordance with the manufacturer's instructions. Briefly, the fungal isolates were incubated in liquid Sabouraud medium (Hangzhou Binhe Microbial Reagent Co., Ltd.) on a shaker at $28^{\circ} \mathrm{C}$ for $24-48 \mathrm{~h}$ until abundant mycelia were observed. Subsequently, the mixture was transferred into a microcentrifuge tube and centrifuged at $25^{\circ} \mathrm{C}$ for $2 \mathrm{~min}$ at
$15,493 \times \mathrm{g}$ to remove the supernatant. Next, the pellet was vortexed first with $300 \mu \mathrm{l}$ of water and then with $900 \mu \mathrm{l}$ of absolute ethanol (Sigma-Aldrich; Merck KGaA). The suspension was then centrifuged at $25^{\circ} \mathrm{C}$ for $2 \mathrm{~min}$ at $15,493 \mathrm{x}$ g to remove the supernatant. The pellet was air dried for $5 \mathrm{~min}$ and then vortexed first with $\sim 10-30 \mu \mathrm{l}$ of $70 \%$ formic acid (Sigma-Aldrich; Merck KGaA) and then with the same amount of acetonitrile. The suspension was then centrifuged for at $25^{\circ} \mathrm{C} 2 \mathrm{~min}$ at $15,493 \mathrm{x} \mathrm{g}$. A total of $1.0 \mu \mathrm{l}$ of the supernatant was added to a 96 -spot polished steel target plate and allowed to dry. A saturated solution of $1.0 \mu \mathrm{l}$ of MALDI-TOF MS matrix (a saturated solution of $\alpha$-cyano-4-hydroxy-cinnamic acid in $50 \%$ acetonitrile and $2.5 \%$ trifluoroacetic acid) was applied to each sample, and the mixture was allowed to dry at room temperature (26-28). Mass spectra were acquired by MALDI-TOF MS and analysed using FlexControl 3.1 and the Bruker filamentous fungi library 1.0 (Bruker Daltonics). The spectrometer was calibrated using the bacterial test standard (BTS; Bruker Daltonics). Each sample was analysed in triplicate. Before each analysis, quality assurance was performed by using Aspergillus fumigatus (ATCC ${ }^{\circledR} 3626^{\mathrm{TM}}$; American Type Culture Collection) and Aspergillus niger (CMCC (F)A3; National Center for Medical Culture Collections) reference strains. Identification scores $\geq 2.000$ indicated species-level identification, scores of 1.700-1.999 indicated genus-level identification, while scores $<1.700$ indicated no identification (29-31). Results showing 'no peaks' required reanalysis.

ITS sequence analysis. Isolates with identification discrepancy between phenotyping and MALDI-TOF MS findings were further analysed by ITS sequencing. A total of 15 filamentous fungi were identified by rDNA-ITS sequence analysis. Fungal genomic DNA was extracted from mycelium with a HiPure Fungal DNA kit (Magen) according to the manufacturer's instructions. The total DNA was amplified by PCR using forward ITS1, 5'-TCCGTAGGTGAACCTGCGG-3' and reverse ITS4, 5'-TCCTCCGCTTATTGATATGC-3' universal primers (32) synthesized by Genewiz, Inc. PCR was performed with the following conditions: An initial denaturation at $95^{\circ} \mathrm{C}$ for $5 \mathrm{~min}$ followed by 35 cycles of $30 \mathrm{sec}$ at $94^{\circ} \mathrm{C}, 30 \mathrm{sec}$ at $52^{\circ} \mathrm{C}$ and $30 \mathrm{sec}$ at $72^{\circ} \mathrm{C}$, with a final extension step at $72^{\circ} \mathrm{C}$ for $5 \mathrm{~min}$. All reactions were performed in $2 \mu \mathrm{l}$ volumes composed of $10 \mathrm{X}$ reaction buffer, $1 \mu \mathrm{l}$ of template, $0.5 \mu \mathrm{l}$ of each dNTP, $0.5 \mu \mathrm{l}$ of each primer, $0.2 \mu \mathrm{l}$ of TransStart Tag DNA Polymerase (Beijing Transgen Biotech Co., Ltd.) and water added to a total volume of $20 \mu \mathrm{l}$. The products were electrophoresed in $1 \%$ agarose gel containing $0.01 \%$ Genecolour $^{\mathrm{TM}}$ nucleic acid stain (Gene Bio Tech Co., Ltd.). All PCR-amplified products were sequenced with an ABI 3730 Sequencer (Applied Biosystems; Thermo Fisher Scientific, Inc.). The ITS sequences of the isolates were compared with reference sequences from GenBank by using the associated BLAST online tool (National Center for Biotechnology Information). Strains were identified on the basis of $99 \%$ similarity or higher.

Antifungal susceptibility testing. Etest gradient strips with fluconazole (range, 0.016-256 $\mu \mathrm{g} / \mathrm{ml}$ ), amphotericin B, voriconazole, itraconazole and caspofungin (range, 0.002-32 $\mu \mathrm{g} / \mathrm{ml}$ ) were purchased from Autobio Diagnostics Co., Ltd. The Etest assay was performed in accordance with the manufacturer's 
Table I. A total of 19 standard strains obtained from China Medical Culture Collection Center.

\begin{tabular}{ll}
\hline Standard strain & Preservation number \\
\hline Aspergillus clavatus & CMCC(F)A4a \\
Aspergillus chevalieri & CMCC(F)A28 \\
Aspergillus flavus & CBS13161 \\
Aspergillus fumigatus & ATCC 3626 \\
Aspergillus nidulans & CMCC(F)A7a \\
Aspergillus niger & CMCC(F)A3 \\
Aspergillus tamarii & CMCC(F)A13 \\
Aspergillus terreus & ATCC 3633 \\
Aspergillus ustus & CMCC(F)A15a \\
Aspergullus versicolor & CBS 245.65 \\
Beauveria bassiana & CMCC(F)B13b \\
Microsporum gypseum & CBS 118893 \\
Paecilomyces lilacinus & CMCC 35539 \\
Penicillium chrysogenum & CMCC(F)B31 \\
Rhizopus microsporus & CMCC(F)B46 \\
Sporothrix schenckii & ATCC 49.12 \\
Talaromyces marneffei & CMCC(F)B33r \\
Trichophyton rubrum & ATCC 4438 \\
Trichophyton tonsurans & CBS 171.65 \\
\hline
\end{tabular}

instructions. Fungi were grown on SDA at $35^{\circ} \mathrm{C}$ for 3-7 day(s) to ensure adequate sporulation from mature fungi. Conidial suspensions were created from harvested cultures, and the turbidity was adjusted to $0.5 \mathrm{McF}$. These suspensions were directly inoculated onto the surface of the antimicrobial test fungal medium (BIO-KONT Co., Ltd.) using swabs, and the plates were incubated at $35^{\circ} \mathrm{C}$ until confluent lawns of filamentous fungi were formed. Subsequently, the surface of each of the plates was dried, and the antifungal agent strips were placed on the plates. The Etest minimum inhibitory concentration (MIC) was recorded as the lowest concentration of an antifungal drug for which the elliptical zone of growth inhibition intersected the Etest strip. Microcolonies within the ellipse were ignored. The Etest MIC was recorded after incubation for 24 or $48 \mathrm{~h}$ at $35^{\circ} \mathrm{C}$. For most fungal species, the final Etest MIC readings were recorded at $24 \mathrm{~h}$ for echinocandins (caspofungin) and at $48 \mathrm{~h}$ for other drugs. Seventy-nine strains were randomly selected and further tested using the Etest MIC method, and quality control for this assay was performed using Candida parapsilosis (ATCC ${ }^{\circledR} 22019^{\mathrm{TM}}$; American Type Culture Collection).

Statistical analysis. Distinct sequences were annotated by Blast search against the NCBI non-redundant database with a cut-off E-value of $10^{-5}$. A low E-value represents high confidence in the annotation.

\section{Results}

Identification of filamentous fungi by phenotypic methods. For most of the evaluated filamentous fungi, the conventional methods take an average of 4 days and cost $\$ 2$ each for completing the identification process. After 3-7 days of incubation, all isolates showed different sizes, shapes, pigments and textures on SDA plates. The phenotypic characteristics of filamentous fungi varied among each other. The Aspergillus species (spp.) grew rapidly and formed loose colonies on SDA plates, with most species exhibiting characteristic colours and shapes. For example, Aspergillus flavus formed fluffy greyish-green colonies consisting predominantly of vegetative hyphae, while $A$. niger usually formed characteristic black colonies on SDA plates. The Penicillium spp. grew more slowly than the Aspergillus spp. On SDA, most Penicillium spp. produced radiated sulcata with a velvety colony surface, and some displayed coloured exudates. For example, Talaromyces marneffei produced a soluble red pigment, which diffused into the agar, lending a red or pink appearance to the reverse side of the colony. Dermatophytes showed radial growth, and many typical isolates of common dermatophytes were identified directly on the SDA plates. Microsporum canis secreted a yellow pigment, and T. rubrum also usually secreted a yellow pigment. Fig. 1 shows 12 typical colony morphologies of filamentous fungi. Of the 123 filamentous fungi analysed in this study, $113(91.9 \%)$ were correctly identified by phenotypic methods, while $5 \mathrm{~T}$. interdigitale, $3 \mathrm{~S}$. globosa and 2 Trichophyton tonsurans were morphologically confused with T. mentagrophytes, S. schenckii and T. rubrum, respectively.

Identification by MALDI-TOF MS. The MALDI-TOF MS method took an average of $15 \mathrm{~min}$ and US\$0.3 for identifying filamentous fungi (starting from the time the culture became available for testing). Of the 123 filamentous fungi analysed by MALDI-TOF MS, 114 (92.70\%) isolates could be identified at the genus level (identification scores, 1.700-1.999), and $80(65.0 \%)$ were correctly identified at the species level (identification scores $\geq 2.000$ ). However, this method failed to identify six strains $(4.9 \%$; scores $<1.700)$. Penicillium chrysogenum and T. mentagrophytes were misidentified as Penicillium camemberti and Trichophytum equinum, respectively (Table II). Fig. 2 shows characteristic MALDI Biotyper spectra of five representative filamentous fungi. These results showed that MALDI-TOF MS rapidly and accurately identified most filamentous fungi and is, therefore, a reliable tool for the diagnosis and treatment of fungal infections.

ITS sequence analysis. Fifteen isolates of filamentous fungi were further identified by rDNA-ITS sequencing analysis. The fungal rDNA ITS were amplified by PCR and then subjected to agarose electrophoresis (Fig. 3). Of the 15 isolates, five were confirmed as T. interdigitale, three as $S$. globosa, two as T. mentagrophytes, two as T.tonsurans, and the remaining as T. marneffei, Aspergillus chevalieri and Beauveria bassiana (Table III). These results indicated that rare or ambiguous fungi were accurately identified by rDNA-ITS sequencing.

Antifungal susceptibility analysis. The results of the quality control tests were within the control limits for the five antifungal drugs. Fluconazole showed the highest MIC that caused $90 \%$ inhibition $\left(\mathrm{MIC}_{90}\right)$ values against the 79 evaluated filamentous fungi: $>256 \mu \mathrm{g} / \mathrm{ml}$ against A. fumigatus, A. flavus, A. niger, T. rubrum, T. tonsurans, Aspergillus nidulans, Aspergillus terreus, Aspergillus tamarii, Aspergillus clavatus, Aspergillus ustus, Aspergillus versicolor and Microsporum 
Table II. Results of identification of filamentous fungi by MALDI-TOF MS ( $n=123)$.

MALDI-TOF MS results, no. (\%)

\begin{tabular}{|c|c|c|c|c|c|}
\hline \multirow[b]{3}{*}{ Strain } & \multirow[b]{3}{*}{$\begin{array}{l}\text { No. of } \\
\text { isolates }\end{array}$} & \\
\hline & & \multicolumn{2}{|r|}{ Unreliable ID } & \multirow[b]{2}{*}{$\begin{array}{c}\text { Genus level } \\
\text { ID only }\end{array}$} & \multirow[b]{2}{*}{$\begin{array}{l}\text { Species } \\
\text { level ID }\end{array}$} \\
\hline & & $\begin{array}{c}\text { No ID } \\
\text { generated }\end{array}$ & $\begin{array}{l}\text { Incorrect ID } \\
\text { (ID generated) }\end{array}$ & & \\
\hline Aspergillus fumigates & 31 & 0 & 0 & 0 & $31(100)$ \\
\hline Aspergillus flavus & 16 & 0 & 0 & 0 & $16(100)$ \\
\hline Aspergillus niger & 7 & 0 & 0 & 0 & $7(100)$ \\
\hline Aspergillus nidulans & 1 & 0 & 0 & 0 & $1(100)$ \\
\hline Aspergillus terreus & 1 & 0 & 0 & 0 & $1(100)$ \\
\hline Aspergillus tamarii & 1 & 0 & 0 & $1(100)$ & 0 \\
\hline Aspergillus clavatus & 1 & 0 & 0 & 0 & $1(100)$ \\
\hline Aspergillus ustus & 1 & 0 & 0 & 0 & $1(100)$ \\
\hline Aspergillus versicolor & 2 & 0 & 0 & 0 & $2(100)$ \\
\hline Trichophyton rubrum & 23 & 0 & 0 & $16(69.6)$ & $7(30.4)$ \\
\hline Epidermophyton floccosum & 5 & 0 & 0 & $5(100)$ & 0 \\
\hline Microsporum canis & 5 & 0 & 0 & $4(80)$ & $1(20)$ \\
\hline Trichophyton tonsurans & 6 & 0 & 0 & $3(50)$ & $3(50)$ \\
\hline Microsporum gypseum & 6 & 0 & 0 & $3(50)$ & $3(50)$ \\
\hline Sporothrix schenckii & 1 & 0 & 0 & $1(100)$ & 0 \\
\hline Sporothrix globosa & 3 & 3 & 0 & 0 & 0 \\
\hline Rhizopus microspores & 1 & 0 & 0 & 0 & $1(100)$ \\
\hline Penicillium chrysogenum & 1 & 0 & 1 (Penicillium camemberti) & 0 & 0 \\
\hline Purpureocillium lilacinus & 1 & 0 & 0 & $1(100)$ & 0 \\
\hline Beauveria bassiana & 1 & 1 & 0 & 0 & 0 \\
\hline Aspergillus chevalieri ${ }^{\mathrm{a}}$ & 1 & 1 & 0 & 0 & 0 \\
\hline Talaromyces marneffe ${ }^{\mathrm{a}}$ & 1 & 1 & 0 & 0 & 0 \\
\hline Trichophyton interdigitale & 5 & 0 & 0 & 0 & $5(100)$ \\
\hline Trichophyton entagrophytes & 2 & 0 & 2 (Trichophytum equinum) & 0 & 0 \\
\hline Total & 123 & $6(4.9)$ & $3(2.4)$ & $34(27.6)$ & $80(65.0)$ \\
\hline
\end{tabular}

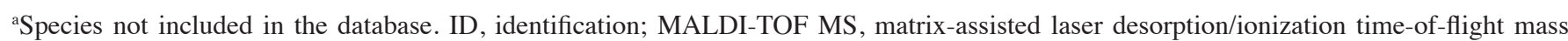
spectrometry.

gypseum; $24 \mu \mathrm{g} / \mathrm{ml}$ against $M$. canis; and $12 \mu \mathrm{g} / \mathrm{ml}$ against Epidermophyton floccosum. By contrast, voriconazole showed the lowest $\mathrm{MIC}_{90}$ values against the 79 filamentous fungi: $\leq 0.38 \mu \mathrm{g} / \mathrm{ml}$ against $A$.fumigatus, $A$. flavus, A. niger, T. rubrum, T. tonsurans, M. canis, E. floccosum, A. nidulans, A. terreus, A. tamarii, $A$. versicolor and $M$. gypseum $; 1.5 \mu \mathrm{g} / \mathrm{ml}$ against A. clavatus; and $2 \mu \mathrm{g} / \mathrm{ml}$ against $A$. ustus. The $\mathrm{MIC}_{90}$ values of caspofungin against the 79 filamentous fungi were the second lowest, next to those of voriconazole: $\leq 1 \mu \mathrm{g} / \mathrm{ml}$ against $A$. fumigatus, A. flavus, A. niger, T. rubrum, T. tonsurans, M. canis, E. floccosum, A. nidulans, A. terreus, and A. tamarii; $6 \mu \mathrm{g} / \mathrm{ml}$ against $M$. gypseum; $8 \mu \mathrm{g} / \mathrm{ml}$ against $A$. ustus and $A$. versicolor; and $12 \mu \mathrm{g} / \mathrm{ml}$ against $A$. clavatus. For T. tonsurans, the $\mathrm{MIC}_{90}$ values of itraconazole corresponded with those of caspofungin. Against E. floccosum, the $\mathrm{MIC}_{90}$ and $\mathrm{MIC}$ that caused $50 \%$ inhibition $\left(\mathrm{MIC}_{50}\right)$ of amphotericin $\mathrm{B}$ both corresponded with those of caspofungin. Against A.fumigatus and T. rubrum, the $\mathrm{MIC}_{90}$ values of amphotericin B corresponded with those of itraconazole. Additionally, the MIC of amphotericin B against
A. terreus and that of itraconazole against both $A$. niger and A. clavatus were $>32 \mu \mathrm{g} / \mathrm{ml}$. The in vitro susceptibilities of the 79 filamentous fungi are summarized in Table IV.

\section{Discussion}

Because of immune deficiency, some individuals, such as those with malignant tumours, HIV infection/acquired immune deficiency syndrome or infectious diseases treated with extended-spectrum antimicrobials as well as transplant recipients, are susceptible to fungal infections $(33,34)$. These infections can be classified as exogenous and endogenous types on the basis of the pathogen source. Candida spp. are still the main pathogens in endogenous opportunistic fungal infections, whereas Aspergillus spp., Cryptococcus spp., dermatophytes, zygomycetes and dimorphic fungi are the predominant sources of exogenous fungal infections $(35,36)$.

At present, identification of fungi, especially filamentous fungi, is very challenging. Conventional methods such as 
A

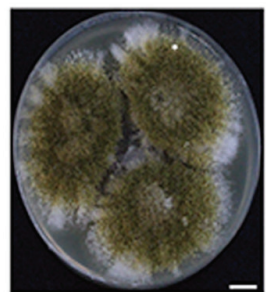

At 3rd day of incubation

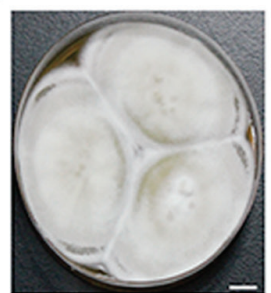

At 5 th day of incubation
B

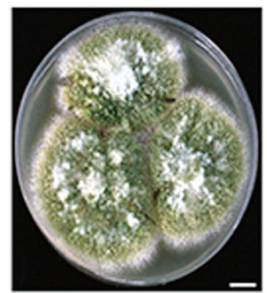

At 3rd day of incubation

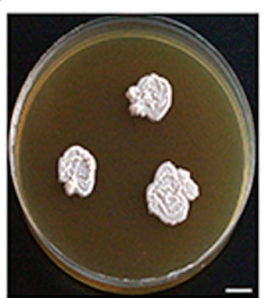

At 7th day of incubation
C

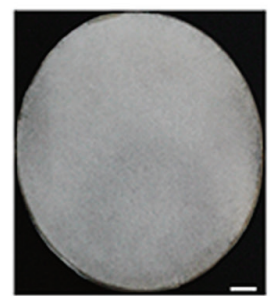

At 3rd day of incubation

I

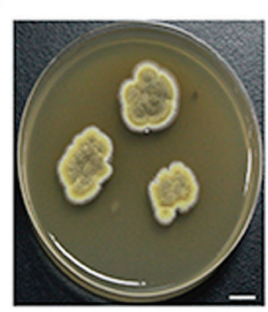

At 7th day of incubation
D

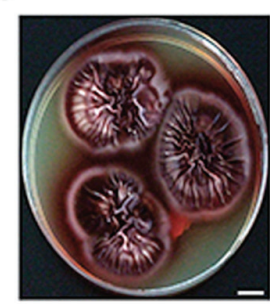

At 5th day of incubation

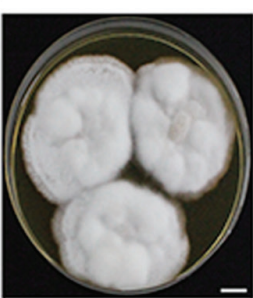

At 7th day of incubation
E

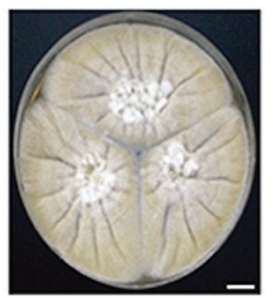

At 5 th day of incubation

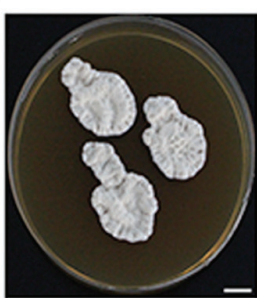

At 7th day of incubation

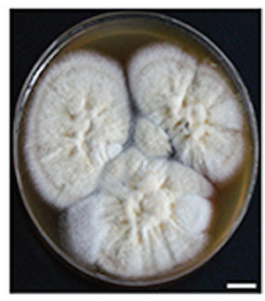

At 5th day of incubation

L

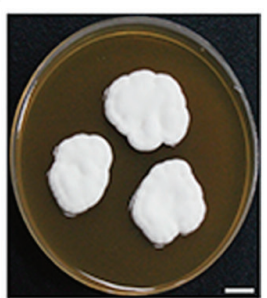

At 7 th day of incubation

Figure 1. Twelve types of colony morphology of filamentous fungi. (A) Aspergillus tamarii (B) Aspergillus flavus, (C) Rhizopus microsporus, (D) Talaromyces marneffei, (E) Aspergillus nidulans, (F) Aspergillus terreus, (G) Aspergillus ustus, (H) Sporothrix schenckii, (I) Aspergillus chevalieri, (J) Trichophyton rubrum, (K) Purpureocillium lilacinus, (L) Beauveria bassiana. Scale bar, $1 \mathrm{~cm}$.

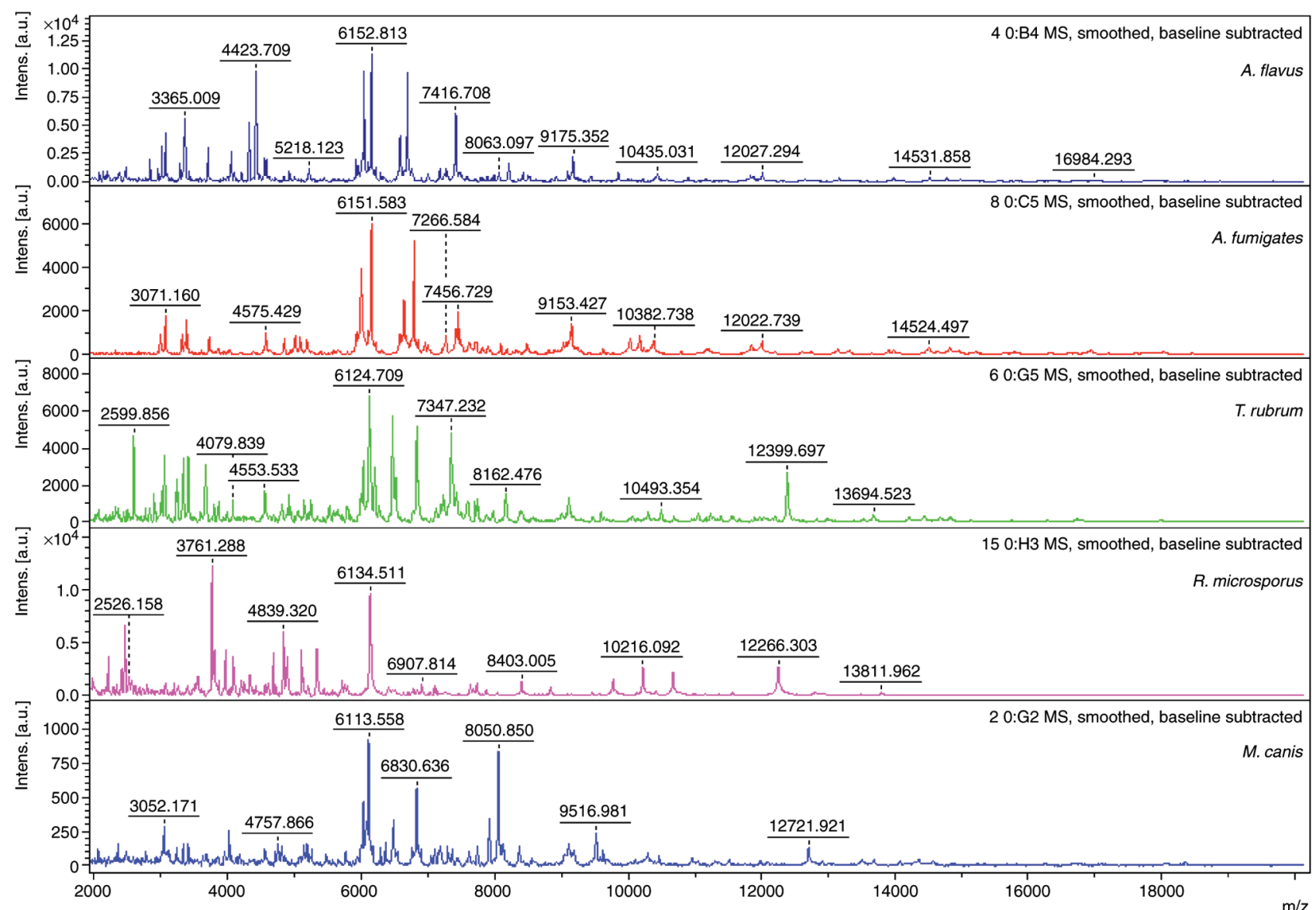

Figure 2. Spectra of Aspergillus flavus, Aspergillus fumigatus, Trichophyton rubrum, Rhizopus microsporus and Microsporum canis generated by the matrix-assisted laser desorption/ionization time-of-flight Bruker Biotyper. The absolute intensities and masses of the ions are shown on the Y and X-axes, respectively. The $\mathrm{m} / \mathrm{z}$ values represent the mass-to-charge ratio. MS, mass spectometry.

macroscopic observation (phenotyping on the basis of growing conditions, colony shape, pigments and texture) and direct or post-staining microscopic examination, are not only time consuming but can also easily misidentify the fungi. Although $\beta-1,3-\mathrm{D}$ glucan and galactomannan tests can rapidly provide evidence of fungal infection, the specificities of these tests 
Table III. Filamentous fungi identified by ITS sequencing analysis $(\mathrm{n}=15)$.

\begin{tabular}{llccrrr}
\hline Strain & \multicolumn{1}{c}{ Result } & Max score & Query cover & E-value & Identity & Accession \\
\hline $134-194 \mathrm{~V}_{\mathrm{o}}$ & Trichophyton interdigitale & 1262 & $100 \%$ & 0.0 & $99 \%$ & KP308373.1 \\
20157639 & T. interdigitale & 1260 & $100 \%$ & 0.0 & $99 \%$ & KP308373.1 \\
$23-20101126$ & Sporothrix globosa & 835 & $99 \%$ & 0.0 & $100 \%$ & JX997737.1 \\
$24-20101149$ & S. globosa & 837 & $99 \%$ & 0.0 & $100 \%$ & JX997737.1 \\
$25-20134489$ & S. globosa & 835 & $99 \%$ & 0.0 & $99 \%$ & LC317795.1 \\
$10-2157180$ & Trichophyton tonsurans & 1249 & $99 \%$ & 0.0 & $99 \%$ & AB220045.1 \\
$130-048 \mathrm{~V}_{\mathrm{o}}$ & T. interdigitale & 1254 & $100 \%$ & 0.0 & $99 \%$ & LC317434.1 \\
$131-104 \mathrm{~V}_{\mathrm{o}}$ & T. interdigitale & 1157 & $100 \%$ & 0.0 & $100 \%$ & $\mathrm{JX} 122224.1$ \\
2157649 & Trichophyton mentagrophytes & 1253 & $99 \%$ & 0.0 & $99 \%$ & KM355551.1 \\
$133-053 \mathrm{~V}_{\mathrm{o}}$ & T. interdigitale & 1266 & $99 \%$ & 0.0 & $100 \%$ & KP308373.1 \\
2157536 & T. mentagrophytes & 1256 & $99 \%$ & 0.0 & $99 \%$ & AB5666303.1 \\
$13-2157226$ & T. tonsurans & 1188 & $99 \%$ & 0.0 & $99 \%$ & AB220045.1 \\
CMCC (f)B13b & Beauveria bassiana & 1042 & $99 \%$ & 0.0 & $99 \%$ & JF429894.1 \\
CMCC (f)B33r & Talaromyces marneffei & 1046 & $100 \%$ & 0.0 & $100 \%$ & KY115196.1 \\
CMCC (f)A28 & Aspergillus chevalieri & 1022 & $100 \%$ & 0.0 & $99 \%$ & KX463363.1
\end{tabular}

ITS, internal transcribed spacer.

are limited because of false negative or false positive results. MALDI-TOF MS has been proven to be a remarkably flexible means for rapid identification and classification of bacteria and yeasts in clinical microbiology (37), and therefore presents a strong challenger to established microscopic and molecular biology methods. However, it has not been widely applied for identifying filamentous fungi because of the complex phylogenetic relationships between species and even more complicated morphology, which can make it difficult to extract fungal proteins.

To the best of the authors' knowledge, there is no standardised procedure for routine identification of filamentous fungi in the clinical laboratory. The limited number of existing studies in this regard have reported varying extraction procedures for fungi (26). Current extraction procedures include bead beating, ultrasonication, and chemical lysis. Here, a simple, economic, and efficient method for extracting filamentous fungi in a clinical laboratory setting has been reported in order to achieve mass spectra of good quality. In the present study, fungi were propagated in liquid cultures and extraction was performed using the ethanol-formic acid method.

In the present study, MALDI-TOF MS achieved $65.0 \%$ (80/123) identification of filamentous fungi at the species level, with a particular high identification rate for Aspergillus spp. (96.8\%; 60/62), which is in agreement with the findings of previous studies $(17,38)$. However, the identification rate of non-Aspergillus spp. was lower; of the 61 non-Aspergillus isolates, only $20(32.8 \%)$ were correctly identified at the species level. The low performance of MALDI-TOF MS in identifying non-Aspergillus spp. may be because of the difficult steps involved in protein extraction. Therefore, it is necessary to identify novel techniques for protein extraction from non-Aspergillus spp. MALDI-TOF MS could not identify some filamentous fungi such as $S$. globosa and T. marneffei, which is likely to be due to their limited reference spectra in the Bruker spectrum database or the highly conserved nature of ribosomal proteins among these species. MALDI-TOF MS also failed to identify other species such as B. bassiana and A. chevalieri because they do not exist in the Bruker spectrum database. Furthermore, the MALDI-TOF Bruker Biotyper generated wrong identifications for P. chrysogenum and T. mentagrophytes. Therefore, it is vital to update the database in order to extend the identification to all fungal species.

The Etest method overcomes the drawback of the BMD method in that the BMD method can be a tedious operation. It also offers other advantages, including direct and easy operation and that it is likely to be available at clinical laboratories that test relatively few filamentous fungi. Consequently, it would be beneficial to validate the Etest method for antifungal susceptibility testing of filamentous fungi. In testing the susceptibility of filamentous fungi against amphotericin B, triazoles and echinocandins, Etest findings show a strong correlation with BMD results (39). In the present study, it was found that incubating the filamentous fungi in RPMI basal medium for 24 or $48 \mathrm{~h}$ was sufficient for visual interpretation of the MIC without cumbersome microscopy.

In the present study, voriconazole (a triazole) showed the most efficient in vitro activity against $A$. ustus, with a MIC of $2 \mu \mathrm{g} / \mathrm{ml}$. However, Lamoth and Alexander (39) have previously reported a voriconazole MIC of $>16 \mu \mathrm{g} / \mathrm{ml}$ against $A$. ustus. In the present study, the antifungal activity of caspofungin was inferior to that of voriconazole, which contradicts the results reported by Shi et al (40). Itraconazole and amphotericin B exhibited slightly higher MIC values against most filamentous fungi except $A$.terreus, A. niger and A. clavatus. The MIC value of amphotericin B against $A$. terreus and those of itraconazole against $A$. niger and A. clavatus were $>32 \mu \mathrm{g} / \mathrm{ml}$, which suggested that the Etest method effectively detected drug-resistant isolates. The MIC of amphotericin B against $A$. terreus in this study $(>32 \mu \mathrm{g} / \mathrm{ml})$ was similar to 


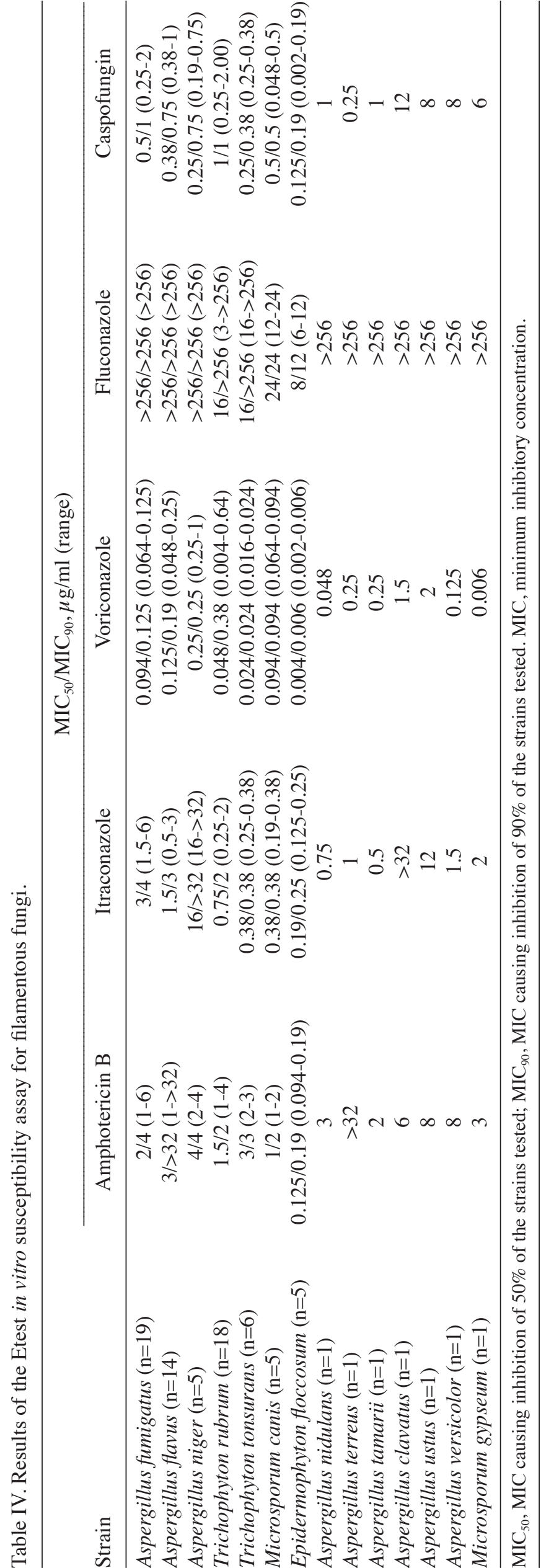

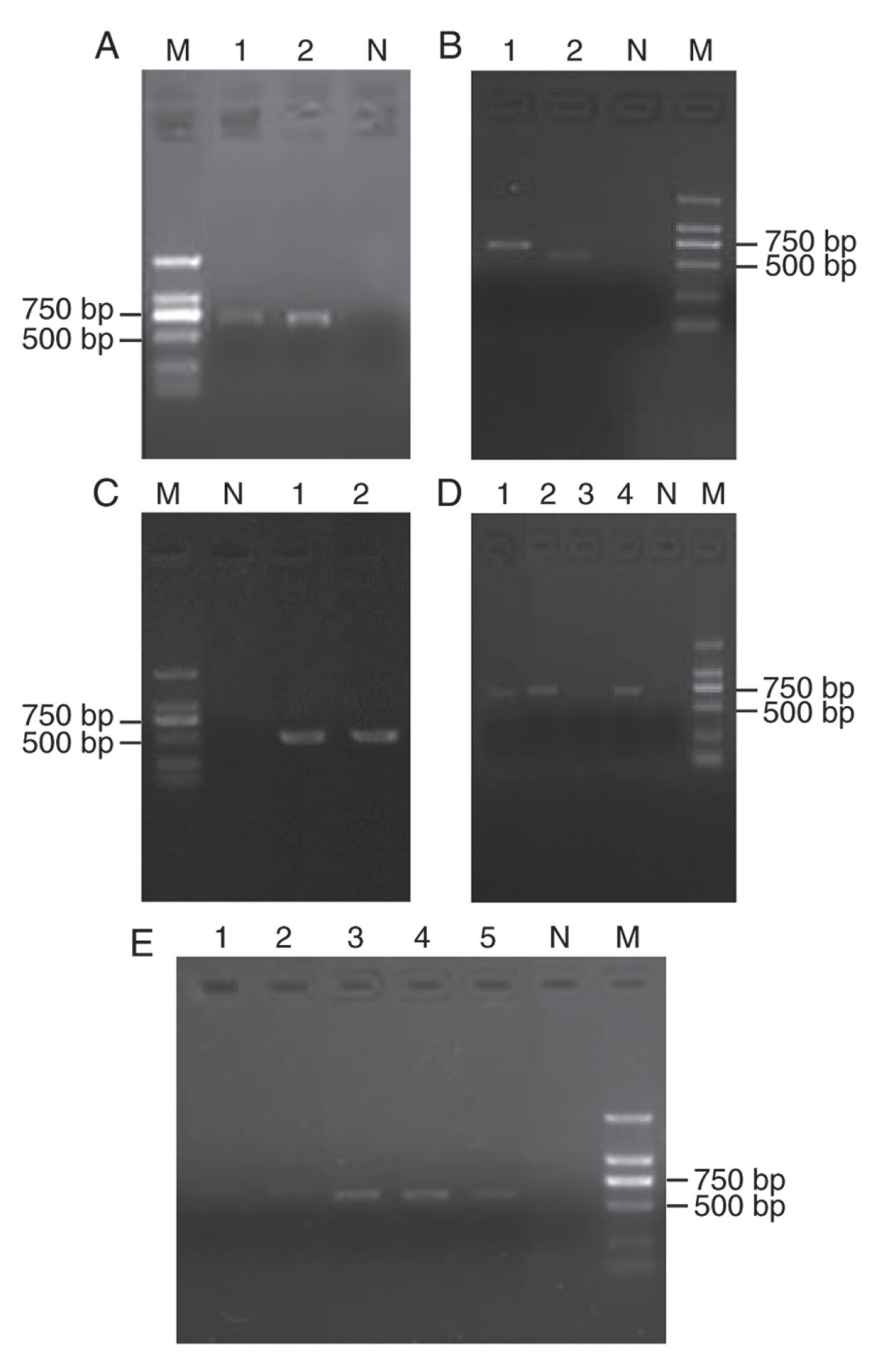

Figure 3. PCR products of the fungal ribosomal DNA internal transcribed spacer sequence. Lane M, Marker. (A) Lane 1, Trichophyton interdigitale; lane 2, Trichophyton mentagrophytes; lane N, negative control. (B) Lane 1, Trichophyton tonsurans; lane 2, Beauveria bassiana; lane N, negative control. (C) Lane 1, Aspergillus chevalieri; lane 2, Talaromyces marneffei; lane $\mathrm{N}$, negative control. (D) Lane 1, T. tonsurans; lane 2, T. interdigitale, lane 3, T. interdigitale; lane 4, T. mentagrophytes; lane N, negative control. (E) Lane 1,T. interdigitale; lane 2, T. interdigitale; lane 3, Sporothrix globosa; lane 4, S. globosa; lane 5, S. globosa; lane N, negative control. bp, base pairs.

that reported in a previous study (41). Furthermore, the data from the present study were similar to the BMD results for the testing of A.terreus against amphotericin B that were found in a study by Vaezi et al (42). A large number of tests have shown that A.terreus is a poor target for amphotericin B and can, therefore, be reported as being amphotericin-B-resistant without further antifungal testing (43).

Fluconazole is by no means the most commonly used antifungal drug for treating filamentous fungi. Although clinical isolates of most filamentous fungi show a broad in vitro resistance to fluconazole, fluconazole is still second-line drugs of choice for prevention and treatment of certain mycoses, including sporotrichosis $(9,44,45)$. Fluconazole showed the highest $\mathrm{MIC}_{50}$ and $\mathrm{MIC}_{90}$ values against all filamentous fungi tested in the present study, which might indicate that the majority of commonly used drugs offer greater resistance than fluconazole. It is interesting to note the activity of azoles against filamentous fungi is variable. Although fluconazole shows 
weak activity against most filamentous fungi, new-generation azoles such as voriconazole might still exhibit favourable activity, highlighting the need for guidance in drug selection. In general, the in vitro activities of voriconazole and caspofungin in the present study were more effective than those of fluconazole, itraconazole and amphotericin $\mathrm{B}$, consistent with previous findings $(39,46)$. On the basis of these observations, the Etest method appears to be suitable for testing the in vitro activity of voriconazole, caspofungin, amphotericin B and itraconazole against filamentous fungi.

In conclusion, it was found that MALDI-TOF MS-based identification of filamentous fungi is less expensive and easier than phenotypic identification and ITS sequence analysis, and its fast turnaround time allows the analysis to be performed more rapidly. As indicated above, it is suitable as a first-line test for identifying filamentous fungi in routine clinical laboratories. However, conventional identification cannot be abandoned and will continue to be an alternative when MALDI-TOF MS fails to provide a definitive result. With the increasing use of MALDI-TOF MS in diagnostic laboratories and the further expansion of online databases of filamentous fungi libraries, a transition to more accurate and rapid identification of filamentous fungi is likely to occur. Etests for direct susceptibility testing for common filamentous fungi has been reported as a rapid antifungal susceptibility testing tool that can provide results in 24-48 h (47). The present in vitro antifungal susceptibility results showed that voriconazole possessed the strongest antifungal activity among the tested drugs and can be used against a broad range of filamentous fungi, while caspofungin possessed better in vitro activity than fluconazole, itraconazole and amphotericin B. Therefore, voriconazole can still be used as the first-line drug for treating serious infections caused by filamentous fungi, while caspofungin could serve as a treatment option for fungal infections. The Etest method is considered an appropriate alternative to guide directed antifungal therapy for routine clinical laboratories. It is important to assess the MIC values of more drugs by comparing clinical outcomes and perfecting interpretive clinical breakpoints, which can help determine whether the use of an antifungal drug is appropriate.

\section{Acknowledgements}

Not applicable.

\section{Funding}

This work was supported by the National Natural Science Foundation of China (grant no. 81572052) and Natural Science Foundation of Jiangsu Province, China (grant no. BK20151178).

\section{Availability of data and materials}

The datasets used and/or analyzed during the current study are available from the corresponding author on reasonable request.

\section{Authors' contributions}

YP performed the experiments and prepared the manuscript. QZ and CX analyzed the data. WS was responsible for study conception and design. All authors read and approved the final manuscript.

\section{Ethics approval and consent to participate}

Not applicable.

\section{Patient consent for publication}

Not applicable.

\section{Competing interests}

The authors declare that they have no competing interests.

\section{References}

1. Kontoyiannis DP, Marr KA, Park BJ, Alexander BD, Anaissie EJ, Walsh TJ, Ito J, Andes DR, Baddley JW, Brown JM, et al: Prospective surveillance for invasive fungal infections in hematopoietic stem cell transplant recipients, 2001-2006: Overview of the Transplant-Associated Infection Surveillance Network (TRANSNET) Database. Clin Infect Dis 50: 1091-1100, 2010.

2. Alcazar-Fuoli L, Mellado E, Alastruey-Izquierdo A, Cuenca-Estrella M and Rodriguez-Tudela JL: Aspergillus section Fumigati: Antifungal susceptibility patterns and sequence-based identification. Antimicrob Agents Chemother 52: 1244-1251, 2008.

3. Grumbt M, Monod M and Staib P: Genetic advances in dermatophytes. FEMS Microbiol Lett 320: 79-86, 2011

4. de Respinis S, Tonolla M, Pranghofer S, Petrini L, Petrini O and Bosshard PP: Identification of dermatophytes by matrix-assisted laser desorption/ionization time-of-flight mass spectrometry. Med Mycol 51: 514-521, 2013.

5. Abastabar M, Mirhendi H, Rezaei-Matehkolaei A, Shidfar MR, Kordbacheh P and Makimura K: Restriction analysis of $\beta$-tubulin gene for differentiation of the common pathogenic dermatophytes. J Clin Lab Anal 28: 91-96, 2014.

6. Cafarchia C, Iatta R, Latrofa MS, Gräser Y and Otranto D: Molecular epidemiology, phylogeny and evolution of dermatophytes. Infect Genet Evol 20: 336-351, 2013.

7. Marimon R, Cano J, Gené J, Sutton DA, Kawasaki M and Guarro J: Sporothrix brasiliensis, S. globosa, and S. mexicana, three new Sporothrix species of clinical interest. J Clin Microbiol 45: 3198-3206, 2007.

8. Nenoff P, Herrmann J and Gräser Y: Trichophyton mentagrophytes sive interdigitale? A dermatophyte in the course of time. J Dtsch Dermatol Ges 5: 198-202, 2007.

9. Gräser Y, Scott J and Summerbell R: The new species concept in dermatophytes-a polyphasic approach. Mycopathologia 166: 239-256, 2008

10. Drogari-Apiranthitou M, Mantopoulou FD, Skiada A, Kanioura L, Grammatikou M, Vrioni G, Mitroussia-Ziouva A, Tsakris A and Petrikkos G: In vitro antifungal susceptibility of filamentous fungi causing rare infections: Synergy testing of amphotericin B, posaconazole and anidulafungin in pairs. J Antimicrob Chemother 67: 1937-1940, 2012.

11. Liu J, Yu Y, Cai Z, Bartlam M and Wang Y: Comparison of ITS and 18S rDNA for estimating fungal diversity using PCR-DGGE. World J Microbiol Biotechnol 31: 1387-1395, 2015.

12. Chen JH, Yam WC, Ngan AH, Fung AM, Woo WL, Yan MK, Choi GK, Ho PL, Cheng VC and Yuen KY: Advantages of using matrix-assisted laser desorption ionization-time of flight mass spectrometry as a rapid diagnostic tool for identification of yeasts and mycobacteria in the clinical microbiological laboratory. J Clin Microbiol 51: 3981-3987, 2013.

13. Del Chierico F, Masotti A, Onori M, Fiscarelli E, Mancinelli L, Ricciotti G, Alghisi F, Dimiziani L, Manetti C, Urbani A, et al: MALDI-TOF MS proteomic phenotyping of filamentous and other fungi from clinical origin. J Proteomics 75: 3314-3330, 2012.

14. Triest D, Stubbe D, De Cremer K, Piérard D, Normand AC, Piarroux R, Detandt M and Hendrickx M: Use of matrix-assisted laser desorption ionization-time of flight mass spectrometry for identification of molds of the Fusarium genus. J Clin Microbiol 53: 465-476, 2015. 
15. Ling $\mathrm{H}$, Yuan $\mathrm{Z}$, Shen J, Wang $\mathrm{Z}$ and $\mathrm{Xu} \mathrm{Y:} \mathrm{Accuracy} \mathrm{of}$ matrix-assisted laser desorption ionization-time of flight mass spectrometry for identification of clinical pathogenic fungi: A meta-analysis. J Clin Microbiol 52: 2573-2582, 2014.

16. Chao QT, Lee TF, Teng SH, Peng LY, Chen PH, Teng LJ and Hsueh PR: Comparison of the accuracy of two conventional phenotypic methods and two MALDI-TOF MS systems with that of DNA sequencing analysis for correctly identifying clinically encountered yeasts. PLoS One 9: e109376, 2014

17. Bille E, Dauphin B, Leto J, Bougnoux ME, Beretti JL, Lotz A, Suarez S, Meyer J, Join-Lambert O, Descamps $\mathrm{P}$, et al: MALDI-TOF MS Andromas strategy for the routine identification of bacteria, mycobacteria, yeasts, Aspergillus spp. and positive blood cultures. Clin Microbiol Infect 18: 1117-1125, 2012.

18. Shokohi T, Aslani N, Ahangarkani F, Meyabadi MF, Hagen F, Meis JF, Boekhout T, Kolecka A and Badali H: Candida infanticola and Candida spencermartinsiae yeasts: Possible emerging species in cancer patients. Microb Pathog 115: 353-357, 2018

19. Aslani N, Janbabaei G, Abastabar M, Meis JF, Babaeian M, Khodavaisy S, Boekhout $\mathrm{T}$ and Badali $\mathrm{H}$ : Identification of uncommon oral yeasts from cancer patients by MALDI-TOF mass spectrometry. BMC Infect Dis 18: 24, 2018.

20. Ranque S, Normand AC, Cassagne C, Murat JB, Bourgeois N, Dalle F, Gari-Toussaint M, Fourquet P, Hendrickx M and Piarroux R MALDI-TOF mass spectrometry identification of filamentous fungi in the clinical laboratory. Mycoses 57: 135-140, 2014.

21. Normand AC, Cassagne C, Ranque S, L'ollivier C, Fourquet P, Roesems S, Hendrickx M and Piarroux R: Assessment of various parameters to improve MALDI-TOF MS reference spectra libraries constructed for the routine identification of filamentous fungi. BMC Microbiol 13: 76, 2013.

22. Lau AF, Drake SK, Calhoun LB, Henderson CM and Zelazny AM: Development of a clinically comprehensive database and a simple procedure for identification of molds from solid media by matrix-assisted laser desorption ionization-time of flight mass spectrometry. J Clin Microbiol 51: 828-834, 2013.

23. Bader O, Weig M, Taverne-Ghadwal L, Lugert R, Gross U and Kuhns M: Improved clinical laboratory identification of human pathogenic yeasts by matrix-assisted laser desorption ionization time-of-flight mass spectrometry. Clin Microbiol Infect 17 $1359-1365,2011$

24. Yonetani S, Ohnishi H, Ohkusu K, Matsumoto T and Watanabe T: Direct identification of microorganisms from positive blood cultures by MALDI-TOF MS using an in-house saponin method. Int J Infect Dis 52: 37-4, 2016.

25. Clinical and Laboratory Standards Institute (CLSI): Reference Method for Broth Dilution Antifungal Susceptibility Testing of Filamentous Fungi; Approved standard. 2nd edition. Clinical and Laboratory Standards Institute, Wayne, PA, M38-A2, 2008.

26. Cassagne C, Ranque S, Normand AC, Fourquet P, Thiebault S, Planard C, Hendrickx M and Piarroux R: Mould routine identification in the clinical laboratory by matrix-assisted laser desorption ionization time-of-flight mass spectrometry. PLoS One 6: e28425, 2011.

27. McMullen AR, Wallace MA, Pincus DH, Wilkey K and Burnham CA: Evaluation of the Vitek MS matrix-assisted laser desorption ionization-time of flight mass spectrometry system for identification of clinically relevant filamentous fungi. J Clin Microbiol 54: 2068-2073, 2016.

28. Normand AC, Cassagne C, Gautier M, Becker P, Ranque S, Hendrickx M and Piarroux R: Decision criteria for MALDI-TOF MS-based identification of filamentous fungi using commercial and in-house reference databases. BMC Microbiol 17: 25, 2017.

29. Becker PT, de Bel A, Martiny D, Ranque S, Piarroux R, Cassagne $C$, Detandt $M$ and Hendrickx M: Identification of filamentous fungi isolates by MALDI-TOF mass spectrometry: Clinical evaluation of an extended reference spectra library. Med Mycol 52: 826-834, 2014

30. Huang Y, Zhang M, Zhu M, Wang M, Sun Y, Gu H, Cao J, Li X, Zhang S, Wang J, et al: Comparison of two matrix-assisted laser desorption ionization-time of flight mass spectrometry systems for the identification of clinical filamentous fungi. World $\mathrm{J}$ Microbiol Biotechnol 33: 142, 2017.

31. Horká M, Kubesová A, Salplachta J, Zapletalová E, Horký J and Slais K: Capillary and gel electromigration techniques and MALDI-TOF MS - suitable tools for identification of filamentous fungi. Anal Chim Acta 716: 155-162, 2012.

32. Tarumoto N, Sakai J, Kodana M, Kawamura T, Ohno H and Maesaki S: Identification of disseminated cryptococcosis using MALDI-TOF MS and clinical evaluation. Med Mycol J 57: E41-E46, 2016.
33. Pagano L, Akova M, Dimopoulos G, Herbrecht R, Drgona L and Blijlevens N: Risk assessment and prognostic factors for mould-related diseases in immunocompromised patients. J Antimicrob Chemother 66 (Suppl 1): i5-i14, 2011

34. Herbrecht R, Bories P, Moulin JC, Ledoux MP and Letscher-Bru V: Risk stratification for invasive aspergillosis in immunocompromised patients. Ann NY Acad Sci 1272: 23-30, 2012.

35. Pemán J, Cantón E, Quindós G, Eraso E, Alcoba J, Guinea J, Merino P, Ruiz-Pérez-de-Pipaon MT, Pérez-del-Molino L, Linares-Sicilia MJ, et al; FUNGEMYCA Study Group: Epidemiology, species distribution and in vitro antifungal susceptibility of fungaemia in a Spanish multicentre prospective survey. J Antimicrob Chemother 67: 1181-1187, 2012.

36. Pana ZD, Farmaki E and Roilides E: Host genetics and opportunistic fungal infections. Clin Microbiol Infect 20: 1254-1264, 2014.

37. Schubert S and Kostrzewa M: MALDI-TOF MS in the Microbiology Laboratory: Current Trends. Curr Issues Mol Biol 23: 17-20, 2017.

38. De CarolisE,PosteraroB,Lass-FlörlC, VellaA,Florio AR, TorelliR, Girmenia C, Colozza C, Tortorano AM, Sanguinetti M, et al: Species identification of Aspergillus, Fusarium and Mucorales with direct surface analysis by matrix-assisted laser desorption ionization time-of-flight mass spectrometry. Clin Microbiol Infect 18: 475-484, 2012.

39. Lamoth F and Alexander BD: Comparing Etest and broth microdilution for antifungal susceptibility testing of the most-relevant pathogenic molds. J Clin Microbiol 53: 3176-3181, 2015.

40. Shi JY, Xu YC, Shi Y, Lü HX, Liu Y, Zhao WS, Chen DM, Xi LY, Zhou X, Wang H, et al: In vitro susceptibility testing of Aspergillus spp. against voriconazole, itraconazole, posaconazole, amphotericin B and caspofungin. Chin Med J (Engl) 123: 2706-2709, 2010.

41. Heo MS, Shin JH, Choi MJ, Park YJ, Lee HS, Koo SH, Lee WG, Kim SH, Shin MG, Suh SP, et al: Molecular identification and amphotericin B susceptibility testing of clinical isolates of Aspergillus from 11 hospitals in Korea. Ann Lab Med 35: 602-610, 2015

42. Vaezi A, Fakhim H, Arastehfar A, Shokohi T, Hedayati MT, Khodavaisy S, Rezaei-Matehkolaei A, Badiee P, Hagen F, Lass-Flörl C, et al: In vitro antifungal activity of amphotericin B and 11 comparators against Aspergillus terreus species complex. Mycoses 61: 134-142, 2018.

43. Arendrup MC, Cuenca-Estrella M, Lass-Flörl C and Hope WW; European Committee on Antimicrobial Susceptibility Testing Subcommittee on Antifungal Susceptibility Testing (EUCAST-AFST): EUCAST technical note on Aspergillus and amphotericin B, itraconazole, and posaconazole. Clin Microbiol Infect 18: E248-E250, 2012.

44. Esquivel BD, Smith AR, Zavrel M and White TC: Azole drug import into the pathogenic fungus Aspergillus fumigatus. Antimicrob Agents Chemother 59: 3390-3398, 2015.

45. Brilhante RS, Rodrigues AM, Sidrim JJ, Rocha MF, Pereira SA, Gremião ID, Schubach TM and de Camargo ZP: In vitro susceptibility of antifungal drugs against Sporothrix brasiliensis recovered from cats with sporotrichosis in Brazil. Med Mycol 54: 275-279, 2016.

46. Gheith S, Saghrouni F, Bannour W, Ben Youssef Y, Khelif A, Normand AC, Piarroux R, Ben Said M, Njah M and Ranque S: In vitro susceptibility to amphotericin $\mathrm{B}$, itraconazole, voriconazole, posaconazole and caspofungin of Aspergillus spp. isolated from patients with haematological malignancies in Tunisia. Springerplus 3: 19, 2014.

47. Pinto E, Lago M, Branco L, Vale-Silva LA and Pinheiro MD: Evaluation of Etest performed in Mueller-Hinton agar supplemented with glucose for antifungal susceptibility testing of clinical isolates of filamentous fungi. Mycopathologia 177: 157-166, 2014.

his work is licensed under a Creative Commons Attribution-NonCommercial-NoDerivatives 4.0 International (CC BY-NC-ND 4.0) License. 